\title{
Genetic Deletion of $C d c 42$ Reveals a Crucial Role for Astrocyte Recruitment to the Injury Site In Vitro and In Vivo
}

\author{
Stefanie Robel, ${ }^{1,2 \star}$ Sophia Bardehle, ${ }^{1,2 \star}$ Alexandra Lepier, ${ }^{1}$ Cord Brakebusch, ${ }^{3}$ and Magdalena Götz ${ }^{1,2}$ \\ ${ }^{1}$ Physiological Genomics, Institute of Physiology, Ludwig-Maximilians University München, 80336 München, Germany, ${ }^{2}$ Institute for Stem Cell Research, \\ HelmholtzZentrum München, 85764 Neuherberg, Germany, and ${ }^{3}$ Biotech Research and Innovation Centre, University of Copenhagen, 2200 Copenhagen, \\ Denmark
}

It is generally suggested that astrocytes play important restorative functions after brain injury, yet little is known regarding their recruitment to sites of injury, despite numerous in vitro experiments investigating astrocyte polarity. Here, we genetically manipulated one of the proposed key signals, the small RhoGTPase Cdc42, selectively in mouse astrocytes in vitro and in vivo. We used an in vitro scratch assay as a minimal wounding model and found that astrocytes lacking Cdc42 (Cdc42 $\Delta$ ) were still able to form protrusions, although in a nonoriented way. Consequently, they failed to migrate in a directed manner toward the scratch. When animals were injured in vivo through a stab wound, $\mathrm{Cdc} 42 \Delta$ astrocytes developed protrusions properly oriented toward the lesion, but the number of astrocytes recruited to the lesion site was significantly reduced. Surprisingly, however, lesions in Cdc $42 \Delta$ animals, harboring fewer astrocytes contained significantly higher numbers of microglial cells than controls. These data suggest that impaired recruitment of astrocytes to sites of injury has a profound and unexpected effect on microglia recruitment.

\section{Introduction}

Astrocytes play crucial roles in the adult brain, yet the molecular mechanisms governing their specific functions are still poorly understood. Throughout the brain astrocytes occupy distinct territories (Bushong et al., 2002; Ogata and Kosaka, 2002), where they perform various functions including the regulation of blood flow in response to neural activity (Iadecola and Nedergaard, 2007; Schummers et al., 2008), requiring contact of their endfeet to blood vessels. Astrocytes are polarized toward the basement membrane around blood vessels and target proteins, such as aquaporin-4 to their endfeet (Bragg et al., 2006). If this interface fails to form properly, as is the case following a loss of $\beta 1$ -

\footnotetext{
Received May 31, 2011; revised July 7, 2011; accepted July 11, 2011.

Author contributions: S.R. and M.G. designed research; S.R. and S.B. performed research; A.L. and C.B. contributed unpublished reagents/analytic tools; S.R. and S.B. analyzed data; S.R., S.B., and M.G. wrote the paper.

This research was supported by grants from the Deutsche Forschungsgemeinschaft (DFG G0 640/7-1, 8-1, 9-1; SPP-1048), including the excellence cluster (enter for Integrated Protein Science Munich, the European Community (Integrated Project EuTRACC, Grant no. LSHG-CT-2007-037445), the SFB 596 and 870, and the Bundesministerium für Bildung und Forschung (Grants 01 GN0503 and FKZ: 01 GN 0820); and the Helmholtz Association in the framework of the "Helmholtz Alliance for Mental Health in Ageing Society" (HELMA), the Impulse \& Networking Fund of the Helmholtz Association (HA-215), the Virtual Institute for Neurodegeneration \& Ageing (VH-VI-252), as well as by the Bavarian research network "ForNeuroCell." We thank foremost the Graduate School of Systemic Neuroscience for financial support of the time-lapse video microscope, without which this study would not have been possible; Alexander Pfeifer (University of Bonn) for the EGFP and Cre-IRES-EGFP lentiviral plasmids; Christian Naumann for cloning the tdTomato and tdTomato-IRES-Cre lentiviral plasmids, as well as for establishment of the time-lapse imaging; Silvia Cappello, Swetlana Sirko, Susan Buckingham, Vishnu Cuddapah, and Harald Sontheimer for critical comments on the manuscript; and Gabi Jäger, Simone Bauer, Andrea Steiner-Mezzadri, Tatiana Simon-Ebert, and Rebecca Krebs for excellent technical assistance.

*S.R. and S.B. contributed equally to this work.

S. Robel's current address: Department of Neurobiology and Center for Glial Biology in Medicine, University of Alabama at Birmingham, Birmingham, AL 35294.

Correspondence should be addressed to Magdalena Götz, Physiological Genomics, Ludwig-Maximilians University München, Pettenkoferstrasse 12, 80336 München, Germany. E-mail: Magdalena.Goetz@helmholtz-muenchen.de.

DOI:10.1523/JNEUROSCI.2696-11.2011

Copyright $\odot 2011$ the authors $\quad 0270-6474 / 11 / 3112471-12 \$ 15.00 / 0$
}

integrins, there results a mild reactive gliosis with all hallmarks of reactive astrogliosis except proliferation (Robel et al., 2009), highlighting the importance of astrocyte polarity. However, little is known about the role of astrocyte polarity after brain injury in vivo.

The reaction of astrocytes to brain injury presents as reactive astrogliosis that ranges from wound closure through astrocyte dedifferentiation, to scar formation (Ridet et al., 1997; Silver and Steindler, 2009; Sofroniew and Vinters, 2010). Astrocyte activation is characterized by hypertrophy and upregulation of many proteins, including the intermediate filaments vimentin and glial fibrillary acidic protein (GFAP), and proteins expressed at earlier developmental stages, such as nestin, Tenascin $\mathrm{C}$ or phosphacan (Buffo et al., 2008; Sirko et al., 2009). Interestingly, following severe injury, a large fraction of reactive astrocytes proliferate and some even regain stem cell potential (Buffo et al., 2008; Robel et al., 2011). While this dedifferentiation may be considered beneficial, reactive astrocytes also upregulate various cell surface molecules, e.g., chondroitin sulfate proteoglycans, and participate in scar formation and inhibition of axon growth across this region (Reier and Houle, 1988; Busch and Silver, 2007). Thus, astrocytes perform numerous functions in response to injury, partially differing depending on the type and size of injury (Pekny and Pekna, 2004; Sofroniew, 2009).

A key aspect common to many injuries is the increase in astrocyte number at the injury site, which has been suggested to be a result of oriented migration and proliferation (Okada et al., 2006; Buffo et al., 2008; Simon et al., 2011). Given that the presence of astrocytes at the injury site is functionally important (Sofroniew, 2009), it is critical to understand the molecular machinery governing astrocyte polarity and recruitment to the injury site in vivo. The small RhoGTPase Cdc42 has emerged as a 
Table 1. First antibodies

\begin{tabular}{llll}
\hline $\begin{array}{l}\text { Recognized } \\
\text { antigen }\end{array}$ & $\begin{array}{l}\text { Post-animal/ } \\
\text { Ig subtype }\end{array}$ & $\begin{array}{l}\text { incubation } \\
\text { conditions }\end{array}$ & Company/Source \\
\hline Cdc42 & Mouse lgG3 & $1: 100$ & Santa Cruz Biotechnology (sc-8401) \\
Cop1 (clone CM1A10) & Mouse lgG1 & $1: 100$ & $\begin{array}{c}\text { Gift from J. E. Rothman, Yale School } \\
\text { of Medicine, New Haven CT }\end{array}$ \\
GFAP & Mouse lgG1 & $1: 1000$ & Sigma (G3898) \\
GFAP & Rabbit & $1: 500$ & Dako/Invitrogen (Z0334) \\
GFP & Mouse lgG1 & $1: 1000$ & Millipore (MAB3580) \\
GFP & Rabbit & $1: 1000$ & Invitrogen (A6455) \\
GFP & Chick & $1: 1000$ & Aves Lab (GFP-1020) \\
Iba1 & Rabbit & $1: 500$ & Wako (019-19741) \\
NeuN & Mouse lgG1 & $1: 100$ & Millipore (MAB377) \\
$\gamma$-Tubulin & Mouse lgG1 & $1: 100$ & Sigma (T5326) \\
pan-Tubulin & Rat & $1: 10$ & Gift from the Department of Anatomy \\
& & & and Cell Biology, Ludwig-Maximilians \\
& & & University München \\
RFP & Rabbit & $1: 500$ & Millipore (AB3216) \\
S100 & Rabbit & $1: 100$ & Sigma (S2644) \\
S100 $\beta$ & Mouse lgG1 & $1: 500$ & Sigma (S2532) \\
\hline
\end{tabular}

key regulator of polarization, influencing directional migration in cultured fibroblasts and astrocytes (Nobes and Hall, 1999; Etienne-Manneville and Hall, 2001, 2003). However, these results were obtained using dominant-negative forms of Cdc42, and genetic deletion of $C d c 42$ in fibroblasts revealed discrepancies in polarization effects and directed migration (Czuchra et al., 2005). This is probably due to inhibition of several other members of the RhoGTPase family by dominant-negative Cdc42 (Czuchra et al., 2005). Therefore, we set out to determine the role of astrocyte polarity by investigating the $\mathrm{Cdc} 42$ function in astrocytes in vitro and in vivo using genetic tools to delete $C d c 42$.

\section{Materials and Methods}

Animals and surgical procedures

C57BL/6J//129/Sv-Cdc42 mice carrying alleles for Cdc42 flanked by loxP sites (Wu et al., 2006) were mated to mice expressing a Cre-recombinase estrogen receptor fusion protein in the GLAST locus (Mori et al., 2006). To label recombined cells, the CAG-CAT-EGFP reporter line, expressing the CMV ( $\beta$-actin promoter) and the loxP flanked chloramphenicol acetyltransferase (CAT) gene upstream of the EGFP cassette (Nakamura et al., 2006) have been used. Mice of either sex were included in the analysis.

All animal procedures were performed in accordance with the Policies on the Use of Animals and Humans in Neuroscience Research, revised and approved by the Society of Neuroscience and the state of Bavaria under license number 55.2-1-54-2531-23/04 or 55.2-1-54-2531-144-07. Tamoxifen was administered as described previously (Mori et al., 2006). For stab wound injury, animals were deeply anesthetized and fixed in a stereotactic frame. Stab wounds were placed into the somatosensory cortex of the right hemisphere (1.5-2 mm long, $0.2 \mathrm{~mm}$ wide and $0.5-0.6$ mm deep).

\section{Histological procedures}

Adult animals were deeply anesthetized and transcardially perfused with PBS followed by $4 \%$ PFA in PBS ( $100 \mathrm{ml} /$ animal). Brains were postfixed in the same fixative for at least $2 \mathrm{~h}$ to maximal overnight at $4^{\circ} \mathrm{C}$, washed in PBS, and embedded in $4 \%$ agarose for cutting $60 \mu \mathrm{m}$ vibratome sections.

For immunofluorescent labeling, sections were incubated overnight at $4^{\circ} \mathrm{C}$ in PBS containing the first antibody, $0.5 \%$ Triton X-100 (TX) and $10 \%$ normal goat serum (NGS), washed in PBS, and incubated for $2 \mathrm{~h}$ at room temperature in $0.5 \%$ TX and $10 \%$ NGS containing the secondary antibody. After washing in PBS, sections were mounted on glass slides and embedded in Aqua-Polymount and covered by a glass coverslip.

Primary and secondary antibodies are listed in Table 1 and Table 2.
Table 2. Secondary antibodies

\begin{tabular}{lllll}
\hline Antibody & Host species & Label & Dilutions & Company \\
\hline$\alpha$-Chick & Goat & Alexa Fluor 488 & $1: 500$ & Invitrogen (A11039) \\
$\alpha$-Chick & Donkey & FITC & $1: 200$ & Dianova (703095155) \\
$\alpha$-Rabbit & Donkey & Alexa Fluor 488 & $1: 500$ & Invitrogen (A21206) \\
& & Cy3 & $1: 500$ & Dianova (711165152) \\
& & Alexa Fluor 594 & $1: 500$ & Invitrogen (A21207) \\
& \multirow{2}{*}{ Goat } & Cy3 & $1: 500$ & Dianova (111165144) \\
& & Biotinylated & $1: 200$ & Vector (BA-1000) \\
$\alpha$-Mouse lgG1 & \multirow{2}{*}{ Goat } & Alexa Fluor 488 & $1: 500$ & Invitrogen (A21121) \\
& & Alexa Fluor 594 & $1: 500$ & Invitrogen (A21125) \\
& & Biotinylated & $1: 200$ & South.B. (1070-08) \\
$\alpha$-Mouse IgG2a & \multirow{2}{*}{ Goat } & Alexa Fluor 488 & $1: 500$ & Invitrogen (A-21131) \\
& & Alexa Fluor 594 & $1: 500$ & Invitrogen (A-21135) \\
$\alpha$-Mouse lgG & \multirow{2}{*}{ Goat } & Alexa Fluor 488 & $1: 500$ & Invitrogen (A11029) \\
& & Cy3 & $1: 500$ & Dianova (115165166) \\
& & Cy5 & $1: 500$ & Dianova (115176072) \\
& \multirow{2}{*}{ Donkey } & Alexa Fluor 594 & $1: 500$ & Invitrogen (A-21203) \\
\hline
\end{tabular}

The terminal deoxynucleotidyl transferase dUTP nick end labeling (TUNEL) assay was performed using an in situ cell death detection kit (Roche) in accordance with the manufacturer's instructions.

The cresyl violet (Nissl) staining was performed as follows. Freefloating vibratome sections were mounted and dried on glass slides before they were dehydrated and washed in xylene two times for $10 \mathrm{~min}$ to remove lipid-rich structures. After rehydration, sections were stained in a $0.1 \%$ cresyl violet solution spiked with acetic acid for $3 \mathrm{~min}$, then washed, dehydrated, and cleared in xylene, before they were embedded in Permount mounting medium and covered by a glass coverslip.

\section{Lentivectors and lentiviral preparation}

Lentiviral expression plasmids contained the sequence for EGFP or CreIRES-EGFP under the CMV promoter (Pfeifer et al., 2001). To avoid any differences in expression levels of the fluorescent proteins, we modified these constructs such that the red fluorescent protein tdTomato was placed directly behind the CMV promoter (LV-CMV-tdTomato and LV-CMVtdTomato-IRES-Cre), thus resulting in comparable signal intensities. To generate the tdTomato-IRES-Cre vector, the Cre-IRES-EGFP plasmid was digested using PstI and SalI to remove the IRES-EGFP cassette. The IRES sequence was amplified with SpeI linkers and placed in front of the Cre sequence into the SpeI restriction site. The tdTomato sequence was then placed behind the CMV promoter by digestion of the CMV-IRES-Cre vector using XbaI, resulting in the lentiviral vector CMV-tomato-IRES-Cre. The tdTomato control construct was generated by replacing the EGFP cassette behind the CMV promoter with the sequence encoding tdTomato.

The lentiviral expression plasmids described above, pCMV $\Delta \mathrm{R} 8.91$ packaging vector (Zufferey et al., 1997), and the pVSVG or pLCMV envelope vector, were cotransfected into 293T cells for production of lentiviral particles as described previously (Naldini et al., 1996). Titers were determined on $293 \mathrm{~T}$ cells, and for most experiments, $8 \times 10^{6}$ viral particles were used per $500 \mu \mathrm{l}$ cell suspension.

\section{In vitro scratch injury assay}

The gray matter of the cerebral cortex from 3-4 postnatal mice $(5-7 \mathrm{~d}$ old) was dissected and mechanically dissociated in Hanks' buffered saline solution containing $10 \mathrm{~mm}$ HEPES. After washing in DMEM medium supplemented with 10\% fetal calf serum, 10 mM HEPES, and Penicillin/ Streptomycin, a single cell suspension was plated into T75 flasks coated with poly-L-ornithine (PLO), and the medium was changed every other day. After reaching confluence, progenitor cells on top of the astrocyte monolayer were removed by thoroughly shaking the cell culture flask, and astrocytes were passaged onto PLO-coated coverslips or directly into PLO-coated 24-well plates for time-lapse experiments. Astrocyte cultures were transduced by the use of lentiviral particles during the splitting step after resuspension of the cells, and directly plated at a density of 70,000 cells per well on plastic or 100,000 cells per well on glass coverslips. Plates were placed into the incubator for $24 \mathrm{~h}$ at $37^{\circ} \mathrm{C}$ and $5 \% \mathrm{CO}_{2}$ before the medium replacement. 

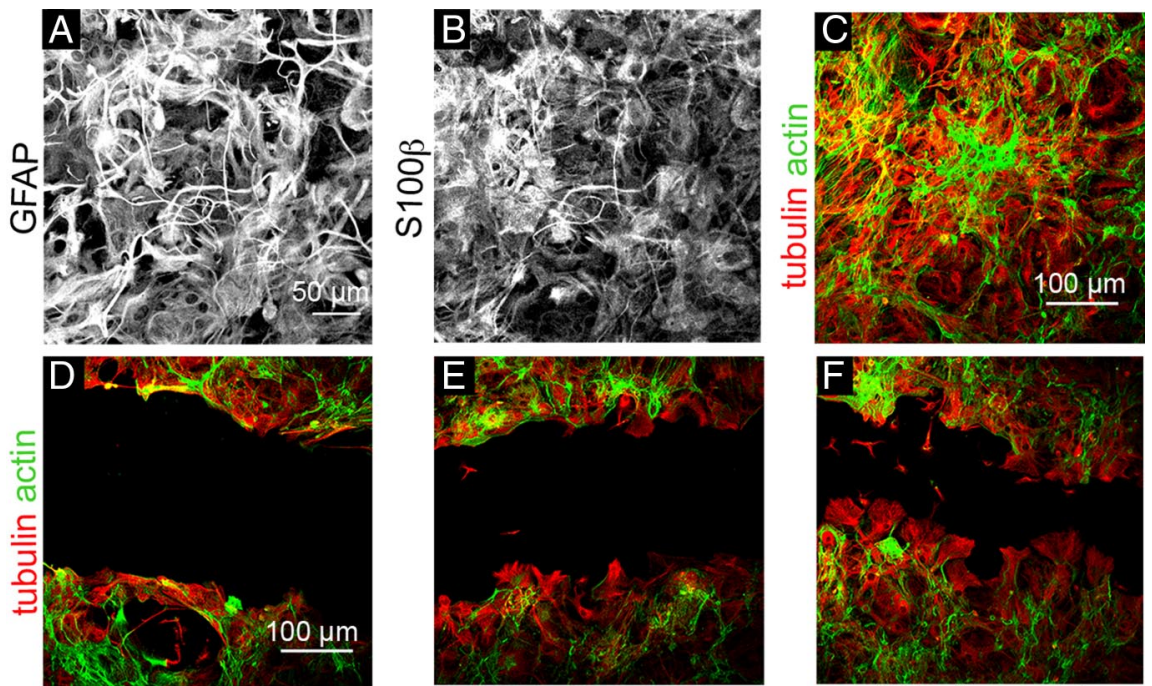

30 min after scratch
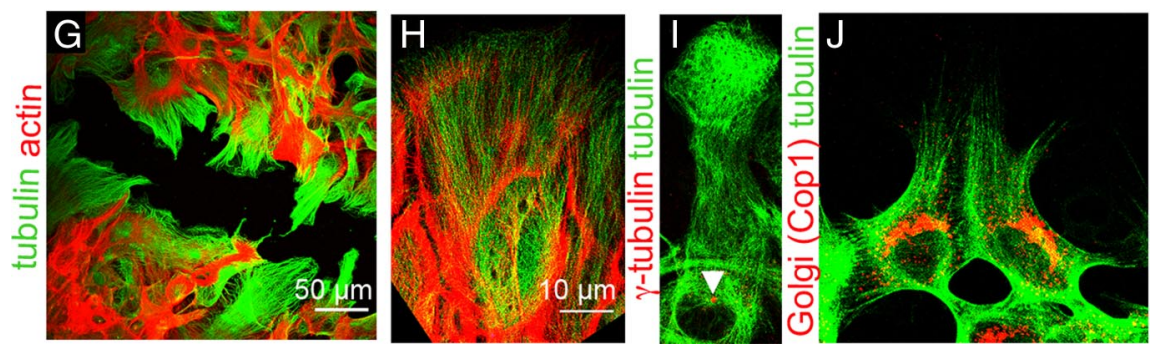

Figure 1. Astrocytes in vitro react to injury by polarization. Postnatal astrocyte cultures were positive for GFAP $(A)$ and/or $S 100 \beta$ $(\boldsymbol{B})$. After scratching, cells in the monolayer $(\boldsymbol{C})$ reacted to the injury and reduced the size of the gap over time $(\boldsymbol{D}-\boldsymbol{F})$. Astrocytes at the scratch formed an extension into the cell-free area $(\mathbf{G} \boldsymbol{J})$. These protrusions were rich in tubulin and stabilized by the actin cytoskeleton $(\boldsymbol{G}, \boldsymbol{H})$. Centrosomes (MTOCS, arrowhead in $\boldsymbol{I}$ ) or Golgi $(\boldsymbol{J})$ of polarized cells were reoriented facing the injury area.

Two weeks later, Cdc42 protein loss could be confirmed by immunocytochemistry exclusively in cells transduced by the lentiviruses containing the Cre recombinase (see Fig. 2).

At the earliest, 2 weeks after viral transduction and 1 week after confluence had been reached, in vitro scratch wound experiments were started following a published protocol (Etienne-Manneville, 2006). Briefly, the confluent astrocyte monolayer was scratched once from the left to the right wall of the well with a sterile $10 \mu$ l plastic tip, resulting in a cell-free cleft $\sim 500 \mu \mathrm{m}$ wide.

For time-lapse experiments, primary astrocyte cultures were prepared from cortices of postnatal $C d c 42^{f l f l}$ mice (postnatal days 5-7, 3-4 animals per experiment) as described above. Two weeks after transduction with tdTomato or tdTomato-IRES-Cre lentivirus, scratch assay experiments combined with video time-lapse microscopy were started. Before scratching the confluent monolayer, Hoechst 33342 dye (Invitrogen) was added to the culture medium at a final concentration of $1 \mu \mathrm{g} / \mathrm{ml}$, and incubated for $20 \mathrm{~min}$ at $37^{\circ} \mathrm{C}$ with $5 \% \mathrm{CO}_{2}$. Cells were washed twice with prewarmed culture medium and scratched $2 \mathrm{~h}$ later. The plate was then placed into the incubation chamber $\left(37^{\circ} \mathrm{C}, 8 \% \mathrm{CO}_{2}\right)$ of an Observer $\mathrm{Zl}$ (Zeiss) fluorescence microscope. Imaging procedures were controlled using AxioVision Rel. 4.7 software for acquisition of phase contrast images every $10 \mathrm{~min}$, and fluorescence images once per hour, for $5 \mathrm{~d}$ using a $20 \times$ objective and an AxioCam HR camera. To control for potential effects due to Cre toxicity, we also transduced astrocytes cultured from WT (C57BL/6) with the Cre-containing virus, and found no signs of toxicity even 2 weeks after transduction. Moreover, changes were observed neither in the orientation of migration toward the scratch nor in the tortuosity.

For analysis of fixed cells, cultures were either immunostained or labeled for actin filaments by phalloidin-Alexa Fluor 488 (Invitrogen) that was added to the secondary antibody solution.

\section{Data analysis}

Results are presented as the mean calculated between different animals (at least three sections per animal and three animals for each time point unless stated differently) or between independent cultures. The variation between animals or cultures is depicted as SEM with one data point representing one animal.

Based on a Gaussian distribution, the data were statistically analyzed by performing an unpaired $t$ test. Means were considered significantly different according to the $p$ value: ${ }^{*} p \leq$ $0.05,{ }^{* *} p \leq 0.01$, and ${ }^{* * *} p \leq 0.001$. Calculations and statistical analysis were done with Excel and GraphPad Prism 3.0, 4.0, or 5.0. Means were considered significantly different as indicated above.

Quantifications after stab wound in vivo. For analysis of astrocyte protrusion formation after a stab wound injury in vivo, lesion size and astrocyte proliferation were assessed using confocal images taken with a Zeiss LSM700 confocal microscope. Length and width of EGFP-positive cells, as well as the longest process toward the stab wound, was measured using ZEN 2008/2009 software (Zeiss). To analyze Nissl+ neuronal number in stab wound lesions, slices were imaged using the Stereo Investigator (mbf Bioscience) software interfaced with an upright Olympus BX-51 microscope. Traces were drawn around regions of interest using a Plan Apo $10 \times$ objective corrected for bright-field observation. Counting was performed using a Plan Apo 40X objective. The Stereo Investigator (mbf Bioscience) software was also used to quantify microglia number in confocal images taken using a Leica SP5 microscope.

Quantifications after scratch wound in vitro. Scratched astrocyte cultures were stained for microtubules that were then observed using a fluorescence microscope (Olympus, BX61) with a $60 \times$ objective. Reorientation of the centrosome [microtubule organizing center (MTOC)] in astrocyte cultures after scratch wound was quantified by separating the area around the nucleus into 4 equal quadrants that joined in the center of the nucleus of the cell of interest. The quadrants were placed with one quadrant facing the scratch and the median of each $90^{\circ}$ angle located either perpendicular or parallel to the scratch. MTOCs were scored as reoriented when they were located in the quadrant facing the scratch. Transduced cells in the first row adjacent to the scratch that displayed one major protrusion three times longer than wide were scored as "protruding cells." The data were obtained from three independent preparations from different litters. For each preparation and time point, two different coverslips and at least 100 transduced cells per coverslip were analyzed and one coverslip was considered a single data point.

Images from time-lapse video microscopy were assembled into a movie and analyzed using the AxioVision Rel. 4.8 software (Zeiss). Quantifications include virus-transduced cells that expressed the red fluorescent protein tomato and lined the front of the scratch. Hoechst labeled nuclei were tracked for three defined time points $(1,3,5 \mathrm{~d})$. The individual tracking paths of every selected cell were used to calculate the following parameters: mean velocity, straight distance, total distance (equals the path length) and tortuosity (equates to the quotient of total distance and straight distance). Protrusion number and transduced cell polarity was assessed $12,24,48$, and 120 h postinjury (p.i.) using red fluorescence images. For protrusion turnover, the presence or absence of each single protrusion was analyzed at a first and a second time point for three different periods $0-24 \mathrm{~h}$ p.i., $24-48$ h p.i. and $48-120$ h p.i. 


\section{Results}

Polarity of astrocytes after scratch injury in vitro

Previous studies used a scratch wound assay after injection of dominant-negative and constitutively active constructs to demonstrate a role for the small RhoGTPase Cdc42 in astrocyte polarity (EtienneManneville and Hall, 2001, 2003; EtienneManneville et al., 2005). In the present study, we used the same assay to examine the effects of $C d c 42$ genetic deletion in astrocytes. Toward this aim, mouse astrocytes were obtained from the postnatal cerebral cortex and grown to full confluence to allow for astrocyte maturation. After 3-4 weeks in culture, cells presented with a flat morphology and could be labeled with antibodies against the astrocyte proteins GFAP (Fig. $1 A$ ) and/or $\mathrm{S} 100 \beta$ (Fig. $1 B$ ). In accordance with previous observations (Etienne-Manneville, 2006), after injuring the monolayer (Fig. $1 C)$, astrocytes extended processes toward the cell-free scratch region and subsequently migrated and populated the scratch over a $24 \mathrm{~h}$ period (Fig. $1 D-F$ ). These scratch-oriented processes had tubulin-positive fibers in the leading tips and were stabilized by the actin cytoskeleton (Fig. $1 G, H$ ) at $24 \mathrm{~h}$ p.i. The formation of protrusions was accompanied by reorientation of both the centrosome (MTOC) labeled by $\gamma$-tubulin (Fig. 1I) and the Golgi apparatus labeled by Cop1 (Fig. 1J) toward the injury site, starting as early as $4 \mathrm{~h}$ after scratch in some cells.

To examine Cdc42 expression in this culture model, astrocytes were stained for $\mathrm{Cdc} 42$ at different time points after monolayer injury (Fig. $2 A-C$ ). Before and shortly after the scratch, endogenous Cdc42 protein was found mainly around the nuclei of astrocytes located at the scratch wound (Fig. 2A), whereas after $8 \mathrm{~h}$, the protein relocalized toward the leading edge of astrocytes facing the scratch (Fig. $2 B, C$ ). This is similar to what has been reported after injecting constructs encoding Cdc42-GFP fusion proteins (Etienne-Manneville and Hall, 2001; Osmani et al., 2010). High-power magnification revealed that Cdc42 was enriched at the tips of newly formed processes (Fig. 2C).

\section{Deletion of Cdc42 reveals a crucial role in orientation of cells toward scratch injury in vitro}

To investigate the role of Cdc42 in astrocyte polarization, we used a genetic deletion designed to avoid potential nonspecific effects of constitutively active and dominant-negative constructs. Postnatal mouse astrocytes containing both alleles of the $C d c 42$ gene flanked by loxP sites (Wu et al., 2006) were cultured and transduced with lentiviruses containing the sequence for either Cre-IRES-EGFP/tdTomato-IRES-Cre (Cdc42 $\Delta$ cultures) or EGFP/ tdTomato alone (control cultures; for control of Cre toxicity, see Materials and Methods). Two weeks after transduction, control and Cdc42 $\Delta$ cultures were stained for Cdc42 (Fig. $\left.2 D-E^{\prime \prime}\right)$. Cre-transduced cells lacked specific staining (Fig. $\left.2 E-E^{\prime \prime}\right)$, thereby confirming that the $C d c 42$ gene was success-
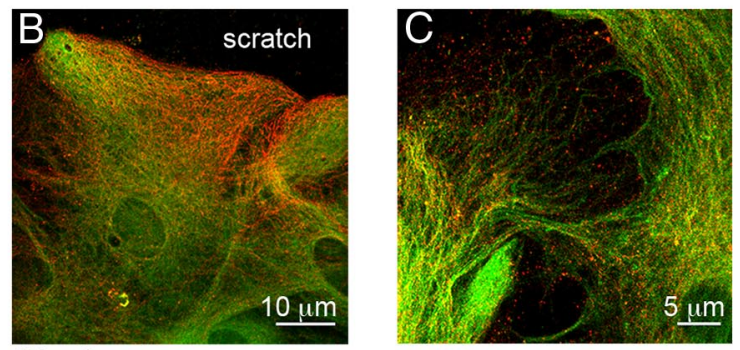

8 h p.i.

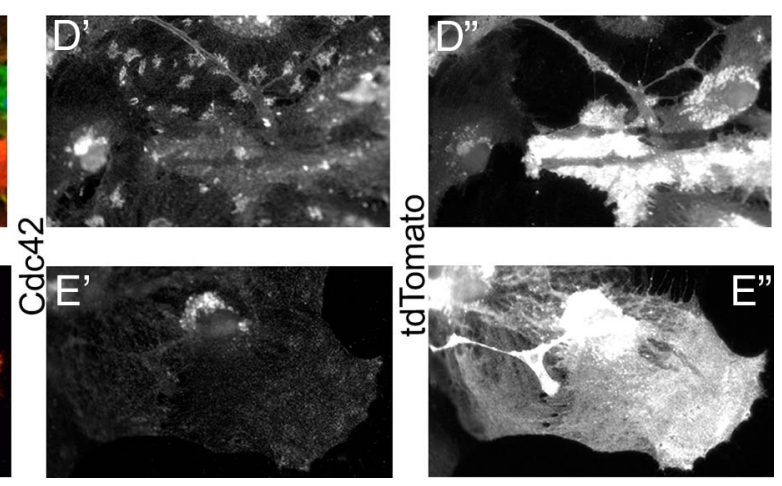

Figure 2. Localization of $\mathrm{Cd}(42$ and protein loss after gene deletion. $A$, $\mathrm{Cdc} 42$ protein is distributed around the nuclei of cells through into the green channel could be observed in control and $\operatorname{Cdc} 42 \Delta$ cultures. Since this effect was also observed in live imaging experiments, it appears to be intrinsic clustering of the tdTomato protein in the Golgi compartment.

fully deleted and Cdc42 protein levels were substantially reduced after lentiviral transduction.

After wounding the confluent astrocyte monolayer (for experimental design, see Fig. $3 A$ ), the reaction of astrocytes was followed in control and Cdc $42 \Delta$ cultures. As expected, most of the astrocytes lining the scratch in control cultures formed long polarized protrusions during the first $24 \mathrm{~h}$ (Fig. $3 B$ ). In contrast, transduced astrocytes in $\mathrm{Cdc} 42 \Delta$ cultures appeared less organized, with multiple protrusions extending randomly from cells (Fig. 3C-E).

To examine the development of this effect more quantitatively, we defined protrusions as (1) cell extensions that were at least three times longer than wide and (2) oriented into the cellfree scratch. We then assessed their appearance at different times after injury. Cells were scored as "unipolar protruding" when they formed a protrusion into the scratch without obvious extensions into other directions. After $30 \mathrm{~min}$, only a small percentage of control- or Cre-transduced astrocytes had formed a protrusion into the scratch $(7 \pm 0.8 \%$ of control-transduced cells with protrusion $0.5 \mathrm{~h}$ p.i., $n$ (cultures) $=6$ ). Over time, an increasing number of control-transduced cells formed protrusions into the cell-free area, and at $24 \mathrm{~h}$ p.i., more than half of the cells were clearly elongated toward the injury site (55.2 $\pm 2.4 \%$ controltransduced cells with protrusion $24 \mathrm{~h}$ p.i., $n=6$ ). In contrast, significantly fewer Cre-transduced Cdc $42 \Delta$ cells formed unipolar protrusions oriented into the scratch at this time $(21.6 \pm 3.0 \%$ Cre-transduced cells with protrusion $24 \mathrm{~h}$ p.i., $n=6, p \leq 0.0001$ ). In addition to this significant reduction of Cdc $42 \Delta$ unipolar cells with scratch oriented protrusions we also noted many Cdc42 $\Delta$ 

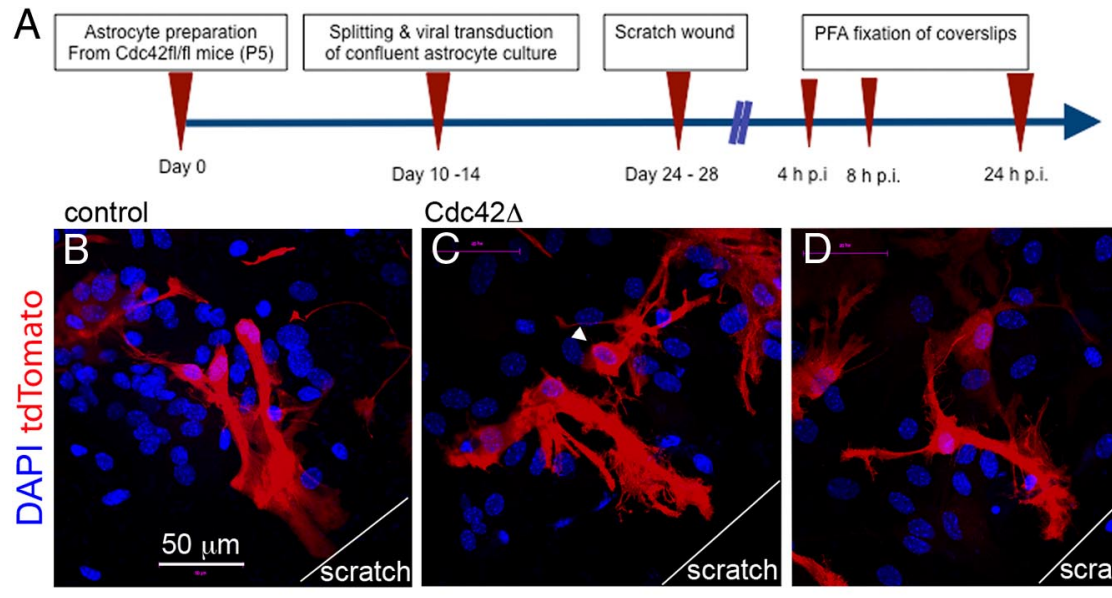

$\mathrm{Cdc} 42 \Delta$
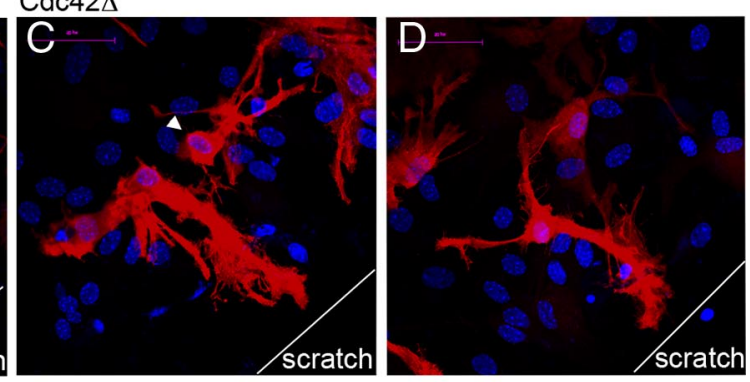

E Number of Protrusions
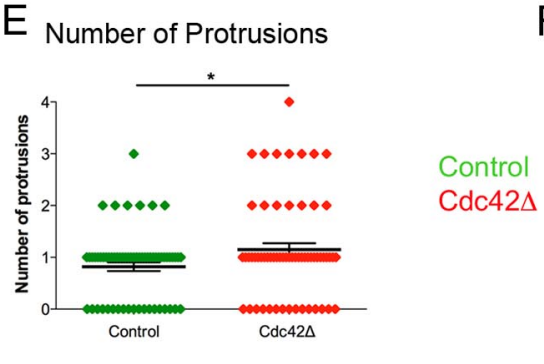

F MTOC reorientation

control
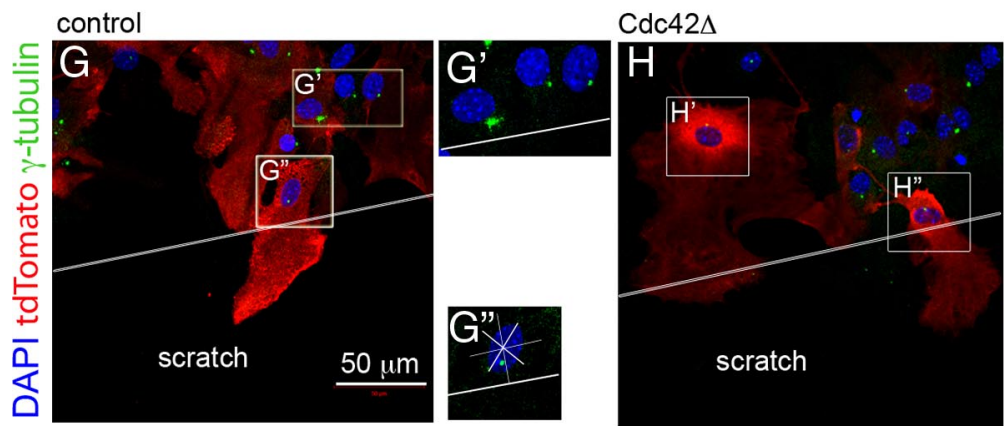

Figure 3. Cdc 42 is involved in orienting protrusions and the MTOC toward a scratch wound in vitro. $A-D$, Astrocytes of postnatal $C d\left(42^{f / f f}\right.$ mice were cultured in a monolayer, transduced with Cre-IRES-tdTomato or tdTomato virus and scratch wounded 2 weeks later $(\boldsymbol{A})$. In control cultures most cells formed long unipolar protrusions toward the direction of the scratch $(\boldsymbol{B})$. $\operatorname{Cdc} 42 \Delta$ cultures were characterized by misoriented cells (white arrowhead in $\boldsymbol{C}$ ) and many cells with multiple protrusions directed into various directions around the cell body $(\boldsymbol{D})$. $\boldsymbol{E}$, Quantification of the number of protrusions per cell is shown (this quantification was done according to the experimental scheme in Fig. $3 A$ at $24 \mathrm{~h} \mathrm{p.i.).} F$, Quantification of MTOC reorientation $24 \mathrm{~h}$ p.i. shows a significant reduction in MTOC reorientation after deletion of $\mathrm{Cd}\left(\mathbf{C} 42 . \mathbf{G}-\boldsymbol{H}^{\prime \prime}\right.$, The majority of first row astrocytes had MTOCs oriented toward the scratch $\left(\boldsymbol{G}, \boldsymbol{G}^{\prime}, \boldsymbol{G}^{\prime \prime}\right)$, whereas MTOCs of Cre-transduced cells were randomly located around the nucleus $\left(\boldsymbol{H}, \boldsymbol{H}^{\prime}, \boldsymbol{H}^{\prime \prime}\right)$. To quantify MTOC orientation, the nucleus was divided into 4 quadrants and MTOCs located in the quadrant facing the scratch were scored as oriented $\left(G^{\prime \prime}\right)$. P5, Postnatal day 5.

unipolar cells with protrusions oriented parallel or even away from the scratch (see example in Fig. $3 C$ ) as well as cells with multiple protrusions (see example in Fig. 3D). Indeed, significantly more Cdc $42 \Delta$ cells had a higher number of protrusions than control cells (Fig. 3E), clearly demonstrating that the reduced number of unipolar cells orienting toward the scratch is not due to a failure of process formation. We therefore asked whether this defect might be due to defects in polarization.

Previously, it has been shown that astrocytes place their MTOC in front of their nucleus toward the direction of a scratch injury, and this appears to be a prerequisite for oriented protrusion formation (Etienne-Manneville and Hall, 2001). To investigate whether the reorientation of the MTOC was disturbed, we used the same assay (Fig. $3 A$ ) and compared the number of re-

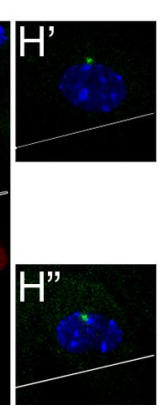

oriented MTOCs in control- and Cretransduced astrocytes (Fig. $\left.3 F-H^{\prime \prime}\right)$. Since the MTOC is located close to the nucleus, the area around the nucleus was separated into 4 equal quadrants and placed such that one quadrant was facing the scratch with each $90^{\circ}$ angle being either perpendicular or parallel to the scratch (Fig. $\left.3 G^{\prime \prime}\right)$. In nonoriented cells, the MTOC should be located randomly around the nucleus, i.e., in $25 \%$ of all cases in any of the 4 quadrants. Only cells with MTOCs clearly belonging to a given nucleus were included in the quantification, and they were scored as reoriented when they were located in the quadrant facing the scratch (Fig. $3 G^{\prime \prime}$ ).

At $30 \mathrm{~min}$ after wounding MTOCs were facing the scratch in a random manner. As soon as $4 \mathrm{~h}$ p.i., some controltransduced astrocytes adjacent to the scratch started to reorient their MTOC toward the scratch (data not shown). This proportion increased even further at $24 \mathrm{~h}$ p.i. (Fig. $3 F, G^{\prime \prime}$ ). Comparable to control cells, at the start of the experiment, MTOCs of Cdc $42 \Delta$ astrocytes were randomly facing the scratch area. However, at $24 \mathrm{~h}$ p.i., the number of reoriented MTOCs within Cdc $42 \Delta$ astrocytes did not increase further (Fig. $3 F, H-H^{\prime \prime}$ ), indicating that $\mathrm{Cdc} 42$ is required for MTOC orientation toward the scratch.

\section{Loss of Cdc42 causes impaired} migration after scratch injury in vitro The above data suggest that $\mathrm{Cdc} 42$ deletion leads to defects in the initial orientation of astrocytes toward the scratch. However, as these data were obtained in fixed cultures, we next used time-lapse video microscopy to observe protrusion formation dynamics in relation to cell migration of virally transduced cells over several days (Fig. 4A).

As expected, control-transduced astrocytes and nontransduced astrocytes adjacent to the scratch formed unipolar protrusions, translocated their cell bodies, and retracted their rear sides to migrate into the scratch. Within $5 \mathrm{~d}$, astrocytes in control cultures had completely closed the 500 $\mu \mathrm{m}$ wide scratch (Fig. $4 B$; Movie 1). However, Cdc $42 \Delta$ astrocytes migrated virtually randomly and were often overtaken by WT cells (Fig. 4B; Movie 2). To clarify the causes for these defects after loss of Cdc42, we examined astrocyte migration and focused on protrusion formation, stability, and orientation, as well as nuclear translocation, as these are all crucial steps in cell migration and scratch wound closure.

Consistent with the data from still analysis described above, protrusion formation per se was not impaired in Cdc $42 \Delta$ astrocytes compared with control cells $(66 \pm 6 \%$ of controltransduced cells and $72 \pm 4 \%$ of Cre-transduced cells formed protrusions 24 h p.i., $n=3$ ), while protrusion orientation was re- 
markably different in $\operatorname{Cdc} 42 \Delta$ astrocytes that had a higher number of protrusions that were randomly oriented compared with control cells (Fig. 3C-E; data not shown). To understand the cause for the increase in protrusion number in $\mathrm{Cdc} 42 \Delta$ astrocytes, we examined protrusion turnover. Within the first $24 \mathrm{~h}$ p.i., protrusion turnover was comparable between Cdc42 $\Delta$ and control astrocytes (Fig. 4C). Thereafter, the number of instable protrusions per cell decreased significantly in control astrocytes, due to stabilization of previously formed protrusions. This was not the case for Cdc42 $\Delta$ astrocyte protrusions, which retained a higher turnover rate at $48 \mathrm{~h}$ p.i. (Fig. $4 C)$. Thus, Cdc $42 \Delta$ astrocytes have difficulties in stable maintenance of protrusions over time.

Since defects in process maintenance may affect migration, we next tracked nuclei of control- or Cre-transduced cells over a period of $5 \mathrm{~d}$ with hourly distance measurement (132 data points) depicted in a tracking path (Fig. 4D). A starting position and an end position was defined for three different time points $(1,3,5 \mathrm{~d}$ p.i.), and based on the fluorescent images taken each hour, the software performed automated tracking. As evidenced by the examples shown in Figure $4 D$, the tracking paths of control astrocytes had a straight linear appearance, whereas the majority of Cdc $42 \Delta$ cells took a rather coiled path (Fig. 4D). Consistent with this impression, the straight distance migrated (shortest path from the starting position to the end position, Fig. $4 E$ ) was significantly reduced for $\mathrm{Cdc} 42 \Delta$ astrocytes to virtually half of the straight distance covered by control cells over the same period (Fig. $4 F$ ). Conversely, the total migration distance, represented by the overall migration distance of a cell including forward, backward, and sideward movements (Fig. 4E), was comparable between control and Cdc42 $\Delta$ astrocytes (Fig. $4 G$ ). Consistent with the equivalent migration distance between control and $\mathrm{Cdc} 42 \Delta$ cells, the average velocity was also not significantly different between control and Cdc $42 \Delta$ cells at 1,3 , and 5 d p.i. (Fig. $4 H$ ). In summary, the overall ability of Cdc $42 \Delta$ cells to migrate was not impaired, but directed migration toward the scratch was aberrant.

If cells migrate the same total distance at the same speed, but cover less straight distance, their migration pathway would likely be rather coiled and curved. This was measured as the tortuosity, the quotient of total and straight distance. An absolute linear movement in one direction (with identical straight and total distance) would have a tortuosity value of 1 . The tortuosity of control-infected astrocytes was $2.5 \pm 0.3$, i.e., their path was 2.5 times longer than a direct route from start to end. Cdc $42 \Delta$ cells

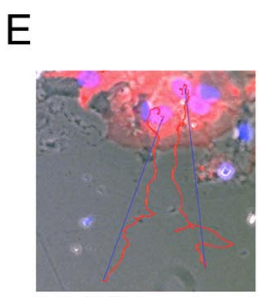

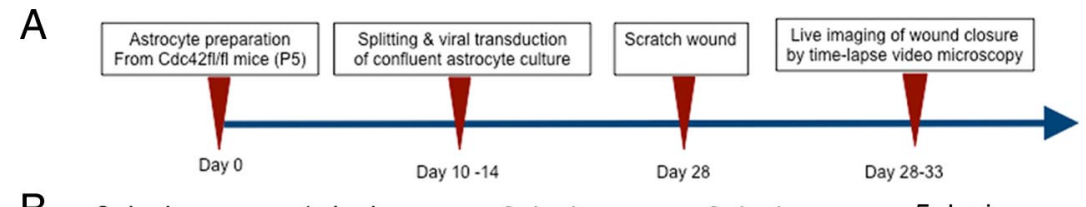
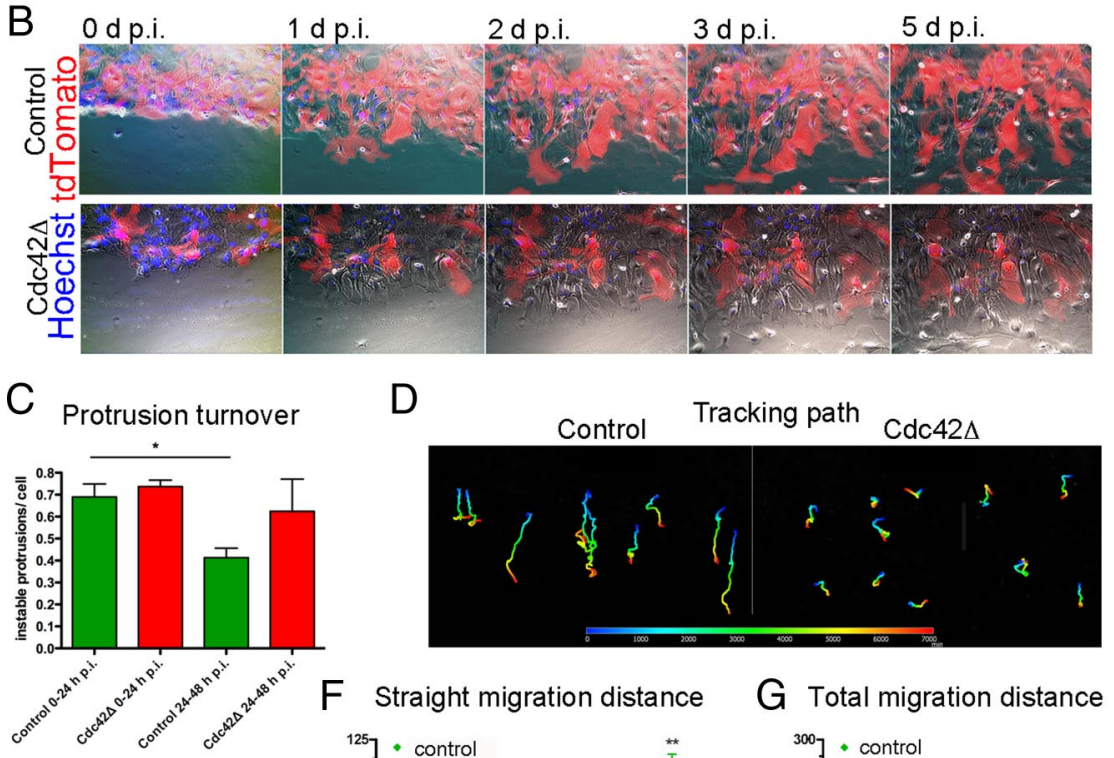

F Straight migration distance

G Total migration distance
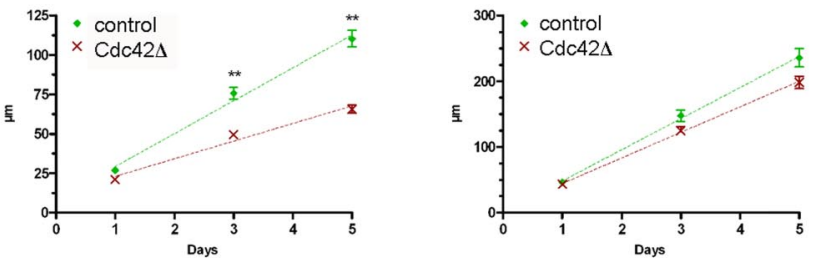

H Migration velocity

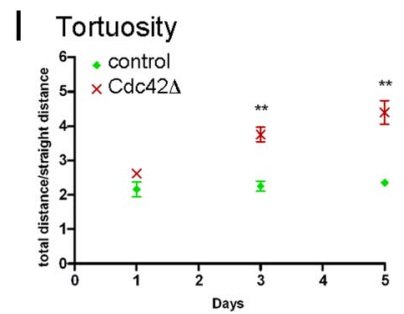

Figure 4. Reactive astrocytes lacking $C d c 42$ show abnormal migration behavior in vitro. $A$, The migratory behavior of $C d c 42^{f / f f l}$ astrocytes transduced with lentiviral particles encoding tdTomato-IRES-Cre or tdTomato alone was analyzed using the in vitro scratch wound assay combined with time-lapse video microscopy. Movie gallery ( 3 channel overlap: phase contrast; blue, Hoechst live dye; red, tdTomato reporter) of the progression of wound closure by scratch-activated astrocytes $0,1,2,3$, and $5 \mathrm{~d}$ p.i. $\boldsymbol{B}$, Nontransduced and control-transduced cells filled the scratch within $5 \mathrm{~d}$, while $\mathrm{Cd}(42 \Delta$ cells showed migration deficits. C, Quantification of the protrusion turnover rate revealed an increase in instable protrusions in $\mathrm{Cd} c 42 \Delta$ cells. $\boldsymbol{D}$, Migration paths recorded by tracking the nuclear translocation over $5 \mathrm{~d}$, show disoriented movements of $\mathrm{Cdc} 42 \Delta$ astrocytes when compared with control cells. $\boldsymbol{E}$, Schematic representation of total migration distance (nuclear path) and straight distance (direct route); both parameters were measured for transduced astrocytes at the scratch 1,3 and $5 \mathrm{~d} \mathrm{p.i.} \boldsymbol{F}-\boldsymbol{H}$, Analysis of the tracking data revealed a reduction of the straight nuclear migration distance in the $C \mathrm{dc}(42 \Delta$ culture $3 \mathrm{~d}$ p.i and later $(\boldsymbol{F})$, but no difference in total migration distance $(\boldsymbol{G})$ and mean velocity $(\boldsymbol{H})$ between control and $\mathrm{Cdc} 42 \Delta$ cells. An increase in tortuosity at 3 and $5 \mathrm{~d}$ p.i. further confirmed the orientation defect of migrating (dc42 $\Delta$ astrocytes $(\boldsymbol{I})$.

exhibited continuously increased tortuosity values from day 1 $(2.6 \pm 0.2)$ to day $5(4.4 \pm 0.6)$ that reached almost double the tortuosity values of control cells (Fig. 4I). Thus, loss of Cdc42 in astrocytes resulted in significantly increased directional changes, despite an overall equal capacity for migration as reflected in the comparable total migration distance and velocity.

The role of $\mathrm{Cdc42}$ in astrocytes at a stab wound injury in vivo These results demonstrate that $\operatorname{Cdc} 42 \Delta$ astrocytes can extend protrusions and migrate at normal speed, but they do so in an undirected manner that ultimately impairs wound closure in 


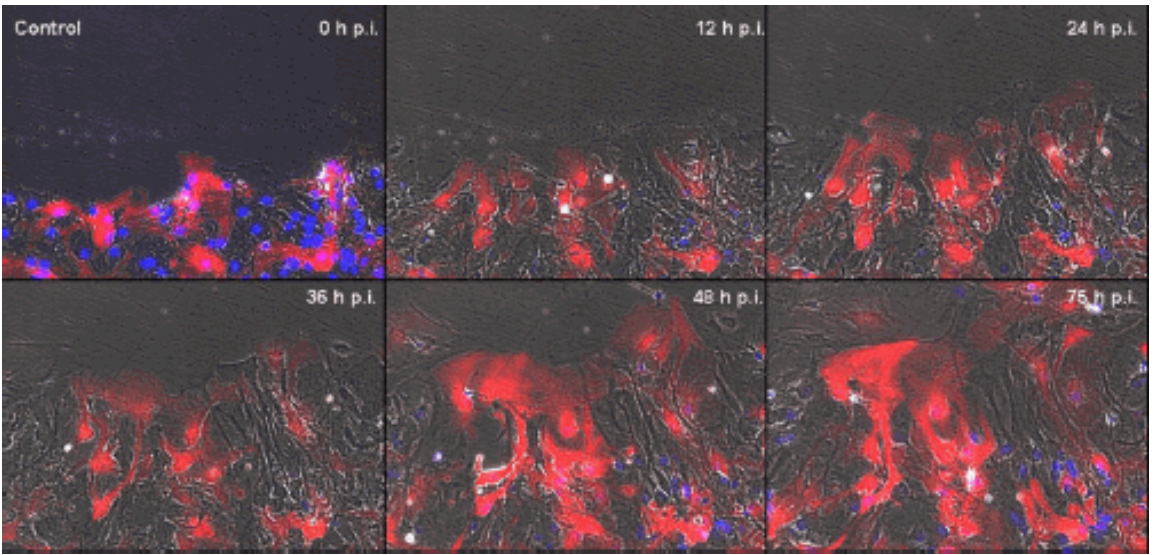

Movie 1. Astrocyte polarization and migration after in vitro scratch wound. Scratch-wounded astrocyte monolayer, followed over $3 \mathrm{~d}$ by time-lapse video microscopy. Nontransduced cells and control-transduced cells (expressing the red fluorescent protein tdTomato) polarize perpendicular to the scratch, and thereafter migrate into the cell-free cleft to fill up the wound.

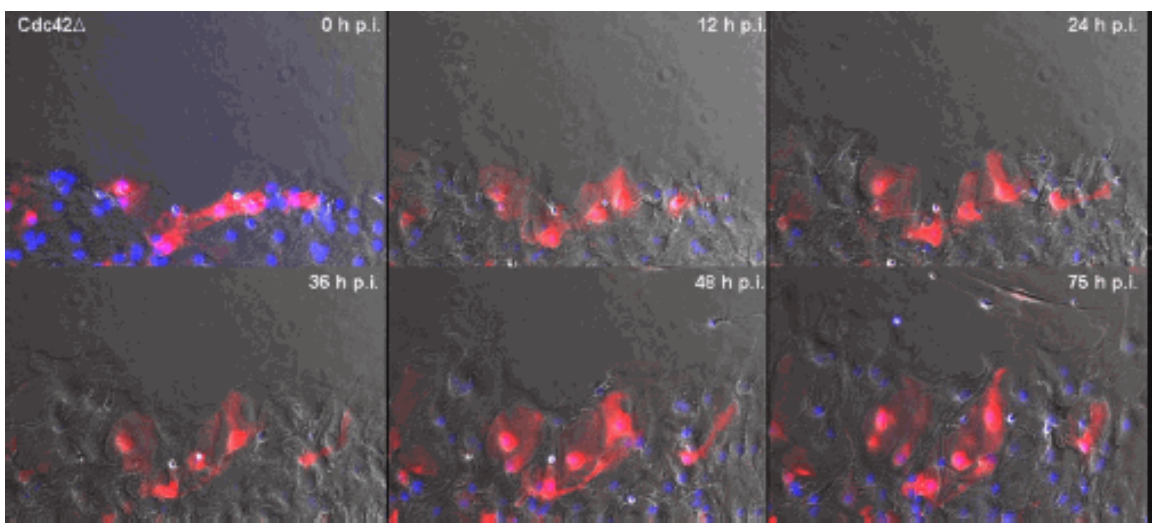

Movie 2. Astrocytes lacking Cdc42 show deficits in polarized migration. After scratching an astrocyte monolayer, $C d c 42$ deficient cells (expressing the red fluorescent protein tdTomato) show impaired scratch-directed polarization and migration. Wild-type cells (cells that do not express tdTomato) bypass impaired Cdc42 $\Delta$ astrocytes.

vitro. This raises the question of whether the defects observed in Cdc42 $\Delta$ astrocytes in the scratch wound assay in vitro can be observed in vivo, where astrocytes react to a complex milieu of signals released by multiple cell types. To examine the behavior of Cdc42 $\Delta$ astrocytes in vivo, we used the stab wound lesion model in the adult mouse cerebral cortex (Buffo et al., 2005, 2008), and monitored the polarity reaction and recruitment of astrocytes toward the site of this acute traumatic injury.

Astrocytes also reacted in vivo to injury by altering their morphology assuming a bipolar shape within $7 \mathrm{~d}$ p.i. (compare Fig. $5 A, B)$. To examine the full morphology of protoplasmic astrocytes beyond their GFAP + processes (for differences between GFAP-immunostaining and fully cytoplasmic extensions, see Wilhelmsson et al., 2006), an EGFP reporter mouse line was crossed to the Tamoxifen-inducible GLAST::CreERT2 line, which allows the induction of genetic recombination in astrocytes (Mori et al., 2006; Buffo et al., 2008). Protoplasmic astrocytes in the gray matter of the cerebral cortex normally possess many fine, radially arranged processes (Fig. 5C). After stab wound injury however, many EGFP-labeled astrocytes became elongated and extended long processes toward the injury border at $7 \mathrm{~d}$ p.i. (Fig. $5 B, D$ ). This reaction was reminiscent of the "palisading zone," a defined region next to the injury core described previously in mouse models of epilepsy (Oberheim et al., 2008). After stab wound, elongated astrocytes were only detected within an approximate area of $200 \mu \mathrm{m}$ around the lesion site, while further away, reactive astrocytes retained their radial symmetry and did not become polarized (Fig. 5E). As observed by GFAP-immunostaining (Fig. 5A,B), also analysis of full morphology revealed that the polarity reaction and formation of the palisading zone developed gradually with few astrocytes beginning to elongate and extending processes toward the injury border at $3 \mathrm{~d}$ p.i., while more than one third of reactive astrocytes proximal to the injury border had an elongated and polar morphology with long processes oriented toward the injury site at $7 \mathrm{~d}$ p.i., Figs. $5 D, 6)$.

To examine the role of $\mathrm{Cdc} 42$ in polarization of astrocytes toward the injury site in vivo, Cdc42 was conditionally deleted in astrocytes in the adult brain using the GLAST::CreERT2 mouse line crossed to the above described line with loxP sites flanking exon 2 of the $C d c 42$ gene. Recombination was achieved by administration of the estrogen analog Tamoxifen to 2- to 3-month-old mice heterozygous for GLAST::CreERT2, positive for the EGFPreporter, and homozygous (referred to as $\operatorname{Cdc} 42 \Delta$ ), heterozygous, or-negative (referred to as control) for the floxed $C d c 42$ allele. Four weeks after Tamoxifen administration, when $\mathrm{Cdc} 42$ protein should be largely gone, a stab wound was placed in the gray matter of the cerebral cortex (Fig. 6A). First, we examined expression of GFAP, an intermediate filament characteristically upregulated in parenchymal astrocytes in response to injury. As expected, a high number of recombined astrocytes close to the injury site expressed GFAP in control animals $(93.5 \pm 2.0$ GFAP + EGFP + cells among EGFP, $n$ (animals) $=3$; Fig. $6 B$ ). After deletion of $\mathrm{Cdc} 42$, a comparable number of astrocytes upregulated GFAP $(95.9 \pm 1.7 \%$ GFAP + EGFP + cells among EGFP in Cdc42 $\Delta, n=3, p=0.43$; Fig. $6 C$ ) and showed a hypertrophic morphology, suggesting that overall injury-induced reactivity was not disturbed by the loss of Cdc42.

Next, we examined the polarization of astrocytes by quantifying cells that had formed an elongated protrusion at $7 \mathrm{~d}$ p.i., when the palisading zone was well established in the control. Accordingly, $39.2 \pm 1.7 \%(n=3)$ of the EGFP + control cells in the palisading zone had formed a protrusion oriented toward the injury (Fig. $6 D$ ). Surprisingly, this number was significantly enhanced in Cdc $42 \Delta$ astrocytes $(68.4 \pm 3.6 \%, n=4, p \leq 0.001$; Fig. $6 E)$. Cdc $42 \Delta$ astrocytes were more elongated $(83.3 \pm 6.8, n=5)$, with a significant increase in total length compared with control astrocytes $(57.6 \pm 6.3 \mu \mathrm{m}$ in control, $n=3, p \leq 0.044$; quantified according to the panel depicted in Fig. $6 F$ ). This was an effect of the stab wound injury, as no differences in astrocyte size or morphology were observed in the contralateral hemispheres (data not shown). Thus, in sharp contrast to the in vitro response, the change toward a bipolar morphology is even more pronounced in astrocytes lacking Cdc42.

In response to injury, astrocyte number increases around the lesion site (Sofroniew and Vinters, 2010). Given that Cdc42- 
deficient astrocytes showed impaired directed migration in vitro, we asked whether astrocyte recruitment toward the injury site in vivo would also be affected. We quantified the number of EGFP+ cells in the hemisphere contralateral to the injury to control for recombination efficiency, and observed an equal number of cells in control and $\mathrm{Cdc} 42 \Delta$ brains (99.7 $\pm 17.8 \%$ of recombined cells in Cdc $42 \Delta$ brains, $n=8$, relative to recombined cell number in control brains, $n=6, p=0.99$ ), demonstrating equal recombination rates. However, within the palisading zone around the stab wound (0-100 $\mu \mathrm{m}$ from the injury core) the number of Cdc $42 \Delta$ EGFP + cells was reduced to less than half $(236.8 \pm 51.1$ cells per $\mathrm{mm}^{2}$ in control, versus $95.5 \pm 9.2$ in Cdc42 $\Delta, n=4, p=0.0347)$, suggesting a severe defect in astrocyte recruitment toward the injury site in the absence of Cdc42.

Astrocyte-specific loss of Cdc42 leads to increased microglia number at the stab wound injury in vivo

Notably, while we observed a strong decrease in the proportion of recombined astrocytes at the injury site, only approximately one third of all astrocytes were recombined in both controls and $\mathrm{fl} / \mathrm{fl}$ mice $(27.5 \pm 2.7 \%$ in control $25.9 \pm 4.8 \%$ in

$\operatorname{Cdc} 42 \Delta, n=3, p=0.78)$. We then considered whether even such a small $15 \%$ decrease in the total population of reactive astrocytes at the injury site might be sufficient to affect other cell types surrounding the injury site. Microglia are the resident immune cells of the brain and are activated and recruited toward injury, most likely interacting with astrocytes throughout reactive gliosis (Hanisch and Kettenmann, 2007). To understand whether the reaction of microglia to injury was changed after loss of Cdc42 in the recombined astrocytes at $7 \mathrm{~d}$ p.i., we quantified Iba1-positive microglia. Contralateral to the injury site, the number of microglia was similar between control and Cdc42 $\Delta$ brains (9023 \pm $1494 \mathrm{Iba} 1+$ cells per $\mathrm{mm}^{3}$ in control and $7916 \pm 665 \mathrm{Iba} 1+$ cells per $\mathrm{mm}^{3}$ in $\operatorname{Cdc} 42 \Delta, p=0.54$; Fig. $\left.7 A, B\right)$. As expected, the number of microglia dramatically increased directly at the lesion (Fig. 7C,D). In the control, microglia number relative to the contralateral hemisphere was approximately fivefold higher at a distance of 100-250 $\mu \mathrm{m}$ from the injury site and tenfold higher directly at the injury site $(0-100 \mu \mathrm{m})$ (Fig. $7 C, E)$. This increase was even more pronounced after astrocyte-specific deletion of $C d c 42$. Here, a 12.5-fold increase in microglia was observed (Fig. $7 D, E ; n=3, p \leq 0.031$ ). Interestingly, the increase in microglia number was observed precisely in the region where astrocyte numbers were decreased (see above), but not at further distant sites (Fig. 7E). Thus, even though only a subset of astrocytes was affected in recruitment to the injury site, these changes were sufficient to affect the microglia reaction.

The proper reaction of astrocytes and microglia postinjury is thought to be essential for protection of the brain from primary neuronal loss. Since both of these cell types are changed after loss of $\mathrm{Cdc} 42$, we next examined neuronal number at the injury site
(Fig. 7F). The pan-neuronal marker NeuN is typically downregulated in neurons surrounding the injury site (data not shown), therefore we used cresyl violet for neuronal somata detection (see red arrow in Fig. 7G,H; Fig. 7G, inset) and compared neuronal cell number in close proximity to the injury site to a similar brain region at $>500 \mu \mathrm{m}$ distant from the injury. Notably, neuron number was reduced to approximately one-third within $100 \mu \mathrm{m}$ around the stab wound at 3 and 7 d p.i. $(n=6$, Fig. $7 F-H)$, but at 100-200 $\mu \mathrm{m}$ distant from the injury, their number was comparable to far distant regions $(93.7 \pm 13.2 \%$ neurons in control, $82.5 \pm 9.4 \%$ neurons in brains with Cdc $42 \Delta$ astrocytes, normalized to neuronal number distal to the injury site, $n=3, p=0.53$ ), indicating a rather concise region of neuronal death in close vicinity to the injury site. In brains with recombined astrocytes depleted of Cdc42 (Fig. $7 G$ ), neuron number was comparably reduced to within $100 \mu \mathrm{m}$ of the injury site at 3 or $7 \mathrm{~d}$ p.i. (Fig. $7 F, H, n=8, p=0.48)$. This is consistent with a comparable number of apoptotic cells detected by TUNEL, 3 d p.i. (9685 \pm 4634 TUNEL cells per $\mathrm{mm}^{3}$ in control brains, $7277 \pm 1490$ TUNEL cells per $\mathrm{mm}^{3}$ in brains with Cdc42 $\Delta$ astrocytes, $n=6$, $p=0.63)$, indicating that primary neuronal death in response to injury is not affected by the modest reduction of astrocyte recruitment achieved by inducible Cdc 42 deletion in $~ 30 \%$ of adult astrocytes.

\section{Discussion}

Here, we demonstrate an essential role for the small RhoGTPase Cdc42 for recruitment of astrocytes to an injury site in vitro and in vivo. While injury-oriented process formation was impaired2 in the absence of Cdc42 in vitro, it appeared normal in vivo. In 
A

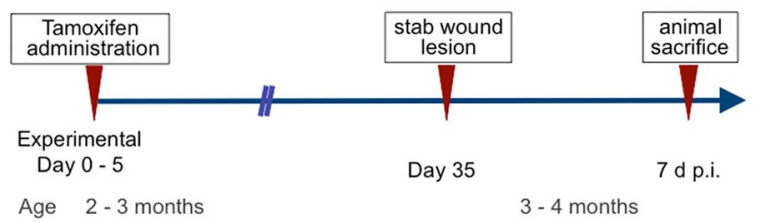

Control
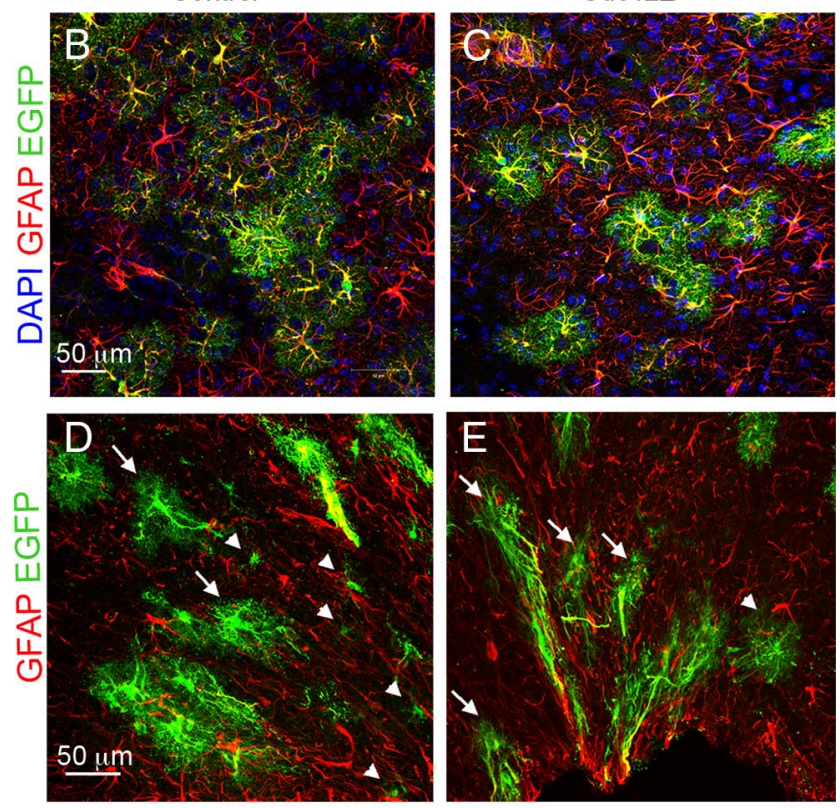

F

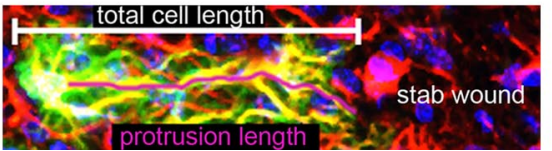

Figure 6. The effects of $c d c 42$ deletion in astrocytes on their morphology at the injury site in vivo. $A$, Genetic recombination was induced in 2- to 3 -month-old animals that were stab wound injured 4 weeks later and killed $7 \mathrm{~d}$.i. following the schedule in $\boldsymbol{A} . \boldsymbol{B}-\boldsymbol{E}$, Astrocytes at the injury site strongly upregulated GFAP in control $(B)$ and $C d c 42 \Delta(C)$ brains. In control brains, $\sim 40 \%$ of recombined EGFP + astrocytes formed a protrusion (white arrows, nonprotruding cells are highlighted by a white arrowhead) within the palisading zone (D). This number was increased in $(\mathrm{dc} 42 \Delta$ animals $(\boldsymbol{E}) . \boldsymbol{F}$, Measurements of protrusion and cell length were done.

contrast, the increase in astrocyte number at the injury site could not be compensated for in vivo. Most importantly, even a modest (based on the recombination frequency of $\sim 30 \%$ ) reduction in astrocyte recruitment to the injury site resulted in a significant increase in microglia number at the injury site, suggesting a crucial role of astrocytes in reducing microglia number at the injury site.

\section{Polarity and migration of astrocytes after injury in vitro}

Scratch injury in vitro is a well established assay used to monitor directed cell migration. Astrocytes in vitro polarize toward a scratch by positioning the centrosome/MTOC between their nucleus and their leading edge and forming directed protrusions before migration into the cell-free scratch (Etienne-Manneville and Hall, 2001, 2003; Etienne-Manneville et al., 2005; Höltje et al., 2005; Etienne-Manneville, 2006; Peng et al., 2008; Ang et al., 2010). Consistent with previous experiments using dominantnegative (Dn)Cdc42, genetic deletion of $C d c 42$ in astrocytes in vitro resulted in MTOC misorientation and a decreased number of cells exhibiting scratch oriented unipolar protrusions, thereby supporting the idea that $\mathrm{Cdc} 42$ affects astrocyte polarity in vitro (Etienne-Manneville and Hall, 2001; Etienne-Manneville, 2008a,b; Li and Gundersen, 2008; Bartolini and Gundersen, 2010). However, we could not confirm all the defects previously observed after DnCdc42 (Etienne-Manneville and Hall, 2001; Czuchra et al., 2005); for example protrusion formation was undisturbed after genetic deletion of Cdc42 in astrocytes in vitro and in vivo. Conversely, Cdc $42 \Delta$ cells often appeared multipolar with multidirectional protrusions around the cell body soon after the scratch. This discrepancy could be due to the dominant-negative constructs affecting other RhoGTPases, since they bind to corresponding guanine nucleotide exchange factors (GEFs) with a higher affinity than endogenous RhoGTPases, preventing effector interaction and subsequent signaling (Feig, 1999). As GEFs are often shared by several RhoGTPase members (Schmidt and Hall, 2002; Rossman et al., 2005), DnCdc42 may also affect Rac1, which is localized to the leading edge of scratch-activated cells by Cdc42-dependent Pak activity, and is responsible for protrusion formation (Cau and Hall, 2005).

Cell migration is governed by the ability to extend, retract, and stabilize membrane protrusions in a defined direction. This can occur in a noncoordinated manner, resulting in random migration, or in a coordinated manner, resulting in directed migration in response to environmental cues (Etienne-Manneville, 2008a). Indeed, tracking Cdc42 $\Delta$ astrocyte nuclei revealed that overall migration was not impaired. However, their tracking paths into the scratch were coiled showing that their directionality was lost. We conclude that in $\operatorname{Cdc} 42 \Delta$ astrocytes an initial polarization defect leads to randomly oriented MTOCs that subsequently cause disoriented movement.

\section{Defects in astrocyte recruitment to the site of brain injury after $\mathrm{Cdc42}$ deletion in astrocytes of the adult brain}

Here, we unravel a hitherto unrecognized role of the small RhoGTPase Cdc42 in astrocyte recruitment to the injury site in vivo, without affecting overall astrocyte reactivity (Okada et al., 2006; Herrmann et al., 2008), since GFAP upregulation and hypertrophic response after injury were normal. Interestingly, in contrast to what has been found in vitro, the polarity reaction of astrocytes in the palisading zone adjacent to the injury site was not impaired by $C d c 42$ deletion, but even enhanced with more cells elongated toward the injury. This discrepancy highlights the limitations of the in vitro scratch assay and the complex nature of cellular interactions and multiple signaling pathways after injury in vivo. While astrocytes in the scratch wound assay are exposed to a cell-free scratch, and almost exclusively astrocyte-released autocrine signals, astrocytes are exposed to a much larger repertoire of signals released from a multitude of cells in vivo, including degenerating neurons, oligodendrocytes and their progenitor cells, the NG2 glia, microglia, and invading cells from the blood system. Indeed, we found that microglia numbers were significantly increased surrounding the stab wound site, thus possibly representing a source of additional signals mediating orientation of palisading astrocytes toward the injury site. Therefore, the in vitro assay is well suited to examine cell-autonomous effects, but extrapolation to the in vivo situation may not always be possible.

Mechanisms controlling Cdc42 activation and localization to the leading edge of the cell are still poorly understood, but ADP ribosylation factor 6 (Arf6)-dependent membrane traffic is such a crucial factor for recruitment of $\mathrm{Cdc} 42$ to the leading edge (Osmani et al., 2010). Moreover, Cdc42 is a downstream effector of integrin signaling (Etienne-Manneville and Hall, 2001; Osmani et al., 2006; Etienne-Manneville, 2008b). Interestingly, interference with $\beta 1$-integrin-mediated signaling at postnatal stages by genetic deletion results in reactive astrogliosis even in 

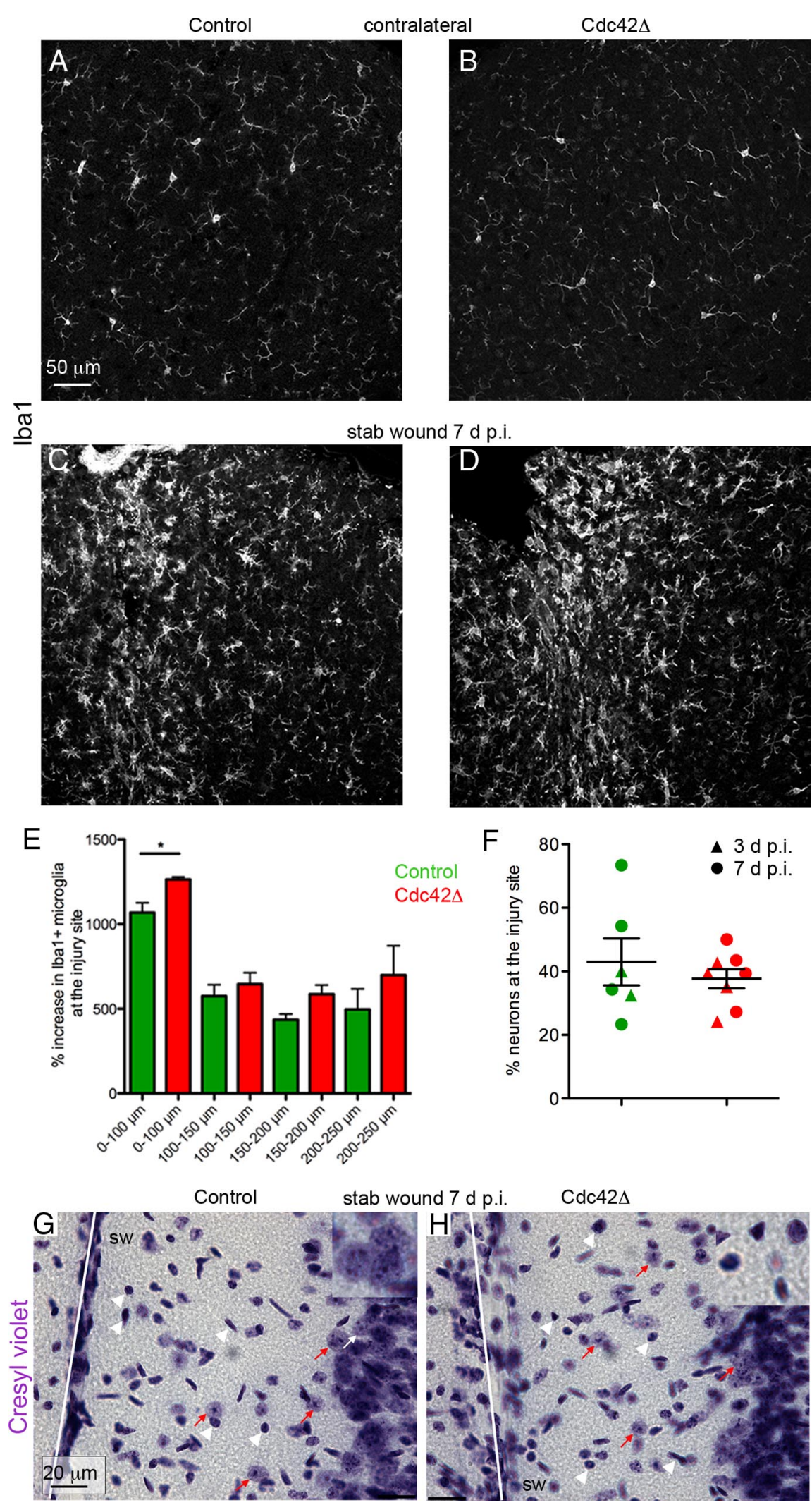

Figure 7. The effects of $C d c 42$ deletion in astrocytes on microglia and neurons at the injury site in vivo. $A-E$, lba1-labeled microglial cells are shown in brains with control $(\boldsymbol{A}, \boldsymbol{B})$ or $\operatorname{Cdc} 42 \Delta(\boldsymbol{C}, \boldsymbol{D})$ astrocytes $7 \mathrm{~d}$ p.i. There were comparable numbers of resting lba1+ microglia in the contralateral hemispheres of control $(\boldsymbol{A})$ and $\operatorname{Cdc} 42 \Delta(B)$ brains. The microglia number significantly increased close to the injury site in brains with control $(\boldsymbol{C})$ or $\operatorname{Cdc} 42 \Delta(\boldsymbol{D})$ astrocytes, but numbers were increased even further after deletion of $C d(42$ in astrocytes $(\boldsymbol{E})$. $\boldsymbol{F}-\boldsymbol{H}$, Neurons were visualized by cresyl violet staining as pale purple cells $(\boldsymbol{G}, \boldsymbol{H}$; indicated by red arrows and enlarged in the inset in $\boldsymbol{G}$ ), and stereotactic counting of these revealed no significant difference after deletion of $(d c 42$ at the injury site $(\boldsymbol{F})$. Neuronal numbers at the injury site were normalized to numbers quantified in a distal unaffected region the uninjured brain in vivo (Robel et al., 2009), and interference with integrin signaling in astrocytes in vitro blocks protrusion formation and polarity (EtienneManneville and Hall, 2001; Osmani et al., 2006; Peng et al., 2008). Notably, in vivo, palisading zone formation and bipolar orientation could also occur in the absence of $\beta 1$-integrins in astrocytes (data not shown), further supporting the concept of alternative pathways in astrocyte orientation in vitro (requiring $\beta 1$-integrins and $\mathrm{Cdc} 42$ ) and in vivo (not requiring either of these). However, other integrins may be compensating in the absence of Cdc42 to mediate effects on astrocyte polarity via other effector pathways (Holly et al., 2000; Lemons and Condic, 2008). For example, $\alpha 6 \beta 4$ integrins interact with intermediate filaments (Rezniczek et al., 1998), which are strongly upregulated after brain injury in astrocytes and may play a key role in stabilizing palisading bipolar astrocytes at the site of injury in vivo. In addition, the basement membrane receptor dystroglycan has been shown to be necessary for astrocyte polarization (Peng et al., 2008), and could act as a redundant mechanism for reactive astrocyte polarization in vivo.

Although Cdc $42 \Delta$ astrocytes were polarized in vivo, the increase in astrocyte number surrounding the injury site was severely impaired, with less than half of the recombined Cdc42-deficient astrocytes found at the injury site. This is not due to developmental defects, as $C d c 42$ was deleted in fully mature astrocytes in the adult brain by Tamoxifen-mediated recombination using GLAST::CreERT2 mice (Mori et al., 2006; Buffo et al., 2008). We therefore conclude that $\mathrm{Cdc} 42$ plays a specific and non-redundant role after brain injury in regulating astrocyte recruitment to the lesion site. Most importantly, recruiting fewer astrocytes to the injury site also affects another cell type as detailed below. It will therefore be important to unravel the precise mechanisms of Cdc42-dependent astrocyte recruitment in vivo. Both directed cell migration and proliferation have been implicated in this process (Okada et al., 2006; Auguste et al., 2007; Buffo et al., 2008; Sofroniew and Vinters, 2010), and only live in vivo imaging will be able to directly determine which of these processes is defective in the absence of Cdc 42 .

(F). Small or shrunken dark purple cells were excluded from the quantitative analysis as they represent glial and/or dying cells (see white arrowheads in $\boldsymbol{H}$ ). sw, Stab wound. 


\section{Consequences of reduced astrocyte recruitment after injury}

Activated astrocytes contribute to scar formation not only by increasing in number, but also by releasing a multitude of molecules, such as chondroitin sulfate proteoglycans, cytokines, and mitogens (Buffo et al., 2010) that act on other cell types. Therefore, a key question was to what extent even a small change in the number of recruited astrocytes may impact other cell types. Indeed, reduction of half of all recombined astrocytes $(\sim 15 \%$ of all astrocytes), resulted in a significant increase in microglia number at the injury site. These observations support quantitative signaling between reactive astrocytes and microglia. Indeed, reactive astrogliosis in the uninjured brain as elicited by $\beta 1$-integrin deletion (Robel et al., 2009) also affected microglial cells, and astrocyte-conditioned medium has been shown to affect the state of microglia activation (Schilling et al., 2001; Kim et al., 2010), consistent with direct signaling from activated astrocytes to microglia. In addition, Cdc42-deficient astrocytes may be defective in their release of signaling molecules due to possible alterations in their secretory activity (Harris and Tepass, 2010). To reveal the precise role of microglia in this context, it will be interesting to investigate whether they are in a "beneficial" state (Thored et al., 2009; Kettenmann et al., 2011) to compensate for the reduction in astrocytes, or whether the increase in microglia is an indicator of an increased detrimental inflammatory reaction due to the defects in Cdc42-deficient astrocytes. Further analysis of reactive astrocytes and microglial cells in this context will be required to determine their exact activation and signaling state. Thus, conditional deletion of $C d c 42$ in astrocytes will serve as a useful model to further study interaction between glial cell types in vivo with the aim of dissecting pathways eliciting the beneficial or adverse roles. Beyond the precise mechanisms, this analysis highlights the key role of reactive astrocytes at the injury site and the profound effect of even small alterations in their number.

\section{References}

Ang SF, Zhao ZS, Lim L, Manser E (2010) DAAM1 is a formin required for centrosome reorientation during cell migration. PLoS One 5:e13064.

Auguste KI, Jin S, Uchida K, Yan D, Manley GT, Papadopoulos MC, Verkman AS (2007) Greatly impaired migration of implanted aquaporin-4deficient astroglial cells in mouse brain toward a site of injury. FASEB J 21:108-116.

Bartolini F, Gundersen GG (2010) Formins and microtubules. Biochim Biophys Acta 1803:164-173.

Bragg AD, Amiry-Moghaddam M, Ottersen OP, Adams ME, Froehner SC (2006) Assembly of a perivascular astrocyte protein scaffold at the mammalian blood-brain barrier is dependent on alpha-syntrophin. Glia 53:879-890.

Buffo A, Vosko MR, Ertürk D, Hamann GF, Jucker M, Rowitch D, Götz M (2005) Expression pattern of the transcription factor Olig2 in response to brain injuries: implications for neuronal repair. Proc Natl Acad Sci U S A 102:18183-18188.

Buffo A, Rite I, Tripathi P, Lepier A, Colak D, Horn AP, Mori T, Götz M (2008) Origin and progeny of reactive gliosis: A source of multipotent cells in the injured brain. Proc Natl Acad Sci U S A 105:3581-3586.

Buffo A, Rolando C, Ceruti S (2010) Astrocytes in the damaged brain: molecular and cellular insights into their reactive response and healing potential. Biochem Pharmacol 79:77-89.

Busch SA, Silver J (2007) The role of extracellular matrix in CNS regeneration. Curr Opin Neurobiol 17:120-127.

Bushong EA, Martone ME, Jones YZ, Ellisman MH (2002) Protoplasmic astrocytes in CA1 stratum radiatum occupy separate anatomical domains. J Neurosci 22:183-192.

Cau J, Hall A (2005) Cdc42 controls the polarity of the actin and microtubule cytoskeletons through two distinct signal transduction pathways. J Cell Sci 118:2579-2587.

Czuchra A, Wu X, Meyer H, van Hengel J, Schroeder T, Geffers R, Rottner K, Brakebusch C (2005) Cdc42 is not essential for filopodium formation, directed migration, cell polarization, and mitosis in fibroblastoid cells. Mol Biol Cell 16:4473-4484.

Etienne-Manneville S (2006) In vitro assay of primary astrocyte migration as a tool to study Rho GTPase function in cell polarization. Methods Enzymol 406:565-578.

Etienne-Manneville S (2008a) Polarity proteins in migration and invasion. Oncogene 27:6970-6980.

Etienne-Manneville S (2008b) Polarity proteins in glial cell functions. Curr Opin Neurobiol 18:488-494

Etienne-Manneville S, Hall A (2001) Integrin-mediated activation of Cdc42 controls cell polarity in migrating astrocytes through PKCzeta. Cell 106:489-498.

Etienne-Manneville S, Hall A (2003) Cdc42 regulates GSK-3beta and adenomatous polyposis coli to control cell polarity. Nature 421:753-756.

Etienne-Manneville S, Manneville JB, Nicholls S, Ferenczi MA, Hall A (2005) Cdc42 and Par6-PKCzeta regulate the spatially localized association of Dlg1 and APC to control cell polarization. J Cell Biol 170:895-901.

Feig LA (1999) Tools of the trade: use of dominant-inhibitory mutants of Ras-family GTPases. Nat Cell Biol 1:E25-E27.

Hanisch UK, Kettenmann H (2007) Microglia: active sensor and versatile effector cells in the normal and pathologic brain. Nat Neurosci 10:1387-1394

Harris KP, Tepass U (2010) Cdc42 and vesicle trafficking in polarized cells. Traffic 11:1272-1279.

Herrmann JE, Imura T, Song B, Qi J, Ao Y, Nguyen TK, Korsak RA, Takeda K, Akira S, Sofroniew MV (2008) STAT3 is a critical regulator of astrogliosis and scar formation after spinal cord injury. J Neurosci 28:7231-7243.

Holly SP, Larson MK, Parise LV (2000) Multiple roles of integrins in cell motility. Exp Cell Res 261:69-74.

Höltje M, Hoffmann A, Hofmann F, Mucke C, Grosse G, Van Rooijen N, Kettenmann H, Just I, Ahnert-Hilger G (2005) Role of Rho GTPase in astrocyte morphology and migratory response during in vitro wound healing. J Neurochem 95:1237-1248.

Iadecola C, Nedergaard M (2007) Glial regulation of the cerebral microvasculature. Nat Neurosci 10:1369-1376.

Kettenmann H, Hanisch UK, Noda M, Verkhratsky A (2011) Physiology of microglia. Physiol Rev 91:461-553.

Kim JH, Min KJ, Seol W, Jou I, Joe EH (2010) Astrocytes in injury states rapidly produce anti-inflammatory factors and attenuate microglial inflammatory responses. J Neurochem 115:1161-1171.

Lemons ML, Condic ML (2008) Integrin signaling is integral to regeneration. Exp Neurol 209:343-352.

Li R, Gundersen GG (2008) Beyond polymer polarity: how the cytoskeleton builds a polarized cell. Nat Rev Mol Cell Biol 9:860-873.

Mori T, Tanaka K, Buffo A, Wurst W, Kühn R, Götz M (2006) Inducible gene deletion in astroglia and radial glia-a valuable tool for functional and lineage analysis. Glia 54:21-34.

Nakamura T, Colbert MC, Robbins J (2006) Neural crest cells retain multipotential characteristics in the developing valves and label the cardiac conduction system. Circ Res 98:1547-1554.

Naldini L, Blömer U, Gallay P, Ory D, Mulligan R, Gage FH, Verma IM, Trono D (1996) In vivo gene delivery and stable transduction of nondividing cells by a lentiviral vector. Science 272:263-267.

Nobes CD, Hall A (1999) Rho GTPases control polarity, protrusion, and adhesion during cell movement. J Cell Biol 144:1235-1244.

Oberheim NA, Tian GF, Han X, Peng W, Takano T, Ransom B, Nedergaard M (2008) Loss of astrocytic domain organization in the epileptic brain. J Neurosci 28:3264-3276.

Ogata K, Kosaka T (2002) Structural and quantitative analysis of astrocytes in the mouse hippocampus. Neuroscience 113:221-233.

Okada S, Nakamura M, Katoh H, Miyao T, Shimazaki T, Ishii K, Yamane J, Yoshimura A, Iwamoto Y, Toyama Y, Okano H (2006) Conditional ablation of Stat 3 or Socs 3 discloses a dual role for reactive astrocytes after spinal cord injury. Nat Med 12:829-834.

Osmani N, Vitale N, Borg JP, Etienne-Manneville S (2006) Scrib controls Cdc42 localization and activity to promote cell polarization during astrocyte migration. Curr Biol 16:2395-2405.

Osmani N, Peglion F, Chavrier P, Etienne-Manneville S (2010) Cdc42 localization and cell polarity depend on membrane traffic. J Cell Biol 191:1261-1269.

Pekny M, Pekna M (2004) Astrocyte intermediate filaments in CNS pathologies and regeneration. J Pathol 204:428-437. 
Peng H, Shah W, Holland P, Carbonetto S (2008) Integrins and dystroglycan regulate astrocyte wound healing: the integrin betal subunit is necessary for process extension and orienting the microtubular network. Dev Neurobiol 68:559-574.

Pfeifer A, Brandon EP, Kootstra N, Gage FH, Verma IM (2001) Delivery of the Cre recombinase by a self-deleting lentiviral vector: efficient gene targeting in vivo. Proc Natl Acad Sci U S A 98:11450-11455.

Reier PJ, Houle JD (1988) The glial scar: its bearing on axonal elongation and transplantation approaches to CNS repair. Adv Neurol 47:87-138.

Rezniczek GA, de Pereda JM, Reipert S, Wiche G (1998) Linking integrin alpha6beta4-based cell adhesion to the intermediate filament cytoskeleton: direct interaction between the beta4 subunit and plectin at multiple molecular sites. J Cell Biol 141:209-225.

Ridet JL, Malhotra SK, Privat A, Gage FH (1997) Reactive astrocytes: cellular and molecular cues to biological function. Trends Neurosci 20:570-577.

Robel S, Mori T, Zoubaa S, Schlegel J, Sirko S, Faissner A, Goebbels S, Dimou L, Götz M (2009) Conditional deletion of betal-integrin in astroglia causes partial reactive gliosis. Glia 57:1630-1647.

Robel S, Berninger B, Götz M (2011) The stem cell potential of glia: lessons from reactive gliosis. Nat Rev Neurosci 12:88-104.

Rossman KL, Der CJ, Sondek J (2005) GEF means go: turning on RHO GTPases with guanine nucleotide-exchange factors. Nat Rev Mol Cell Biol 6:167-180.

Schilling T, Nitsch R, Heinemann U, Haas D, Eder C (2001) Astrocyte-released cytokines induce ramification and outward $\mathrm{K}+$ channel expression in microglia via distinct signalling pathways. Eur J Neurosci 14:463-473.

Schmidt A, Hall A (2002) Guanine nucleotide exchange factors for Rho GTPases: turning on the switch. Genes Dev 16:1587-1609.

Schummers J, Yu H, Sur M (2008) Tuned responses of astrocytes and their influence on hemodynamic signals in the visual cortex. Science 320:1638-1643.

Silver DJ, Steindler DA (2009) Common astrocytic programs during brain development, injury and cancer. Trends Neurosci 32:303-311.

Simon C, Götz M, Dimou L (2011) Progenitors in the adult cerebral cortex: cell cycle properties and regulation by physiological stimuli and injury. Glia 59:869-881.

Sirko S, Neitz A, Mittmann T, Horvat-Bröcker A, von Holst A, Eysel UT, Faissner A (2009) Focal laser-lesions activate an endogenous population of neural stem/progenitor cells in the adult visual cortex. Brain 132:2252-2264.

Sofroniew MV (2009) Molecular dissection of reactive astrogliosis and glial scar formation. Trends Neurosci 32:638-647.

Sofroniew MV, Vinters HV (2010) Astrocytes: biology and pathology. Acta Neuropathol 119:7-35.

Thored P, Heldmann U, Gomes-Leal W, Gisler R, Darsalia V, Taneera J, Nygren JM, Jacobsen SE, Ekdahl CT, Kokaia Z, Lindvall O (2009) Longterm accumulation of microglia with proneurogenic phenotype concomitant with persistent neurogenesis in adult subventricular zone after stroke. Glia 57:835-849.

Wilhelmsson U, Bushong EA, Price DL, Smarr BL, Phung V, Terada M, Ellisman MH, Pekny M (2006) Redefining the concept of reactive astrocytes as cells that remain within their unique domains upon reaction to injury. Proc Natl Acad Sci U S A 103:17513-17518.

Wu X, Quondamatteo F, Lefever T, Czuchra A, Meyer H, Chrostek A, Paus R, Langbein L, Brakebusch C (2006) Cdc42 controls progenitor cell differentiation and beta-catenin turnover in skin. Genes Dev 20:571-585.

Zufferey R, Nagy D, Mandel RJ, Naldini L, Trono D (1997) Multiply attenuated lentiviral vector achieves efficient gene delivery in vivo. Nat Biotechnol 15:871-875. 